\title{
NEARLY KÄHLERIAN EMBEDDINGS OF SYMPLECTIC MANIFOLDS*
}

\author{
DAVID BORTHWICK ${ }^{\dagger}$ AND ALEJANDRO URIBE ${ }^{\ddagger}$
}

1. Introduction and statement of results. Let $X, \omega$ be a $2 n$-dimensional compact symplectic manifold without boundary. There always exists a compatible almost-complex structure $J$ on $X$, i.e.

$$
\omega(J u, J v)=\omega(u, v), \quad \omega(\cdot, J \cdot) \gg 0 .
$$

Let $g$ denote the associated Riemannian metric on $X$, that is $g(u, v)=\omega(u, J v)$. Assume that $\omega$ defines an integral cohomology class, which means that there exists a complex line bundle, $L \rightarrow X$, with a connection $\nabla$ whose curvature is $-i \omega .^{1}$

Using the notion of "generalized Szegö projectors" [8], one can "quantize" $(X, \omega, J)$ by a suitable sequence of finite-dimensional Hilbert spaces, $\mathcal{H}_{k} \subset C^{\infty}\left(X, L^{\otimes k}\right)$, defined for all $k$ sufficiently large. (We'll recall their definition in the next section.) The usual procedure of algebraic geometry yields well-defined maps,

$$
F_{k}: X \longrightarrow \mathbb{P H}_{k}^{*}
$$

The dimension of $\mathcal{H}_{k}$ is the Riemann-Roch polynomial of $X$ evaluated at $k$, that is

$$
\operatorname{dim} \mathcal{H}_{k}=\int e^{k[\omega]} \tau=\operatorname{Vol}(X) k^{n}+O\left(k^{n-1}\right),
$$

where $\tau$ is the Todd class.

In this paper we study the asymptotic geometry of the maps $F_{k}$. We propose the general philosophy that the quantization $\left\{\mathcal{H}_{k}\right\}$ is a natural object that can be used to study the geometry of $(X, \omega)$. The main results are as follows:

THEOREM 1.1. Let $\Omega_{k}$ and $g_{k}^{0}$ denote respectively the symplectic form and Kähler metric of $\mathbb{P H}_{k}^{*}$. Then there exist positive constants $C_{1}$ and $C_{2}$ and $k_{0}$ such that for all $k>k_{0}$

$$
\forall x \in X \quad\left|\frac{1}{k} F_{k}^{*} \Omega_{k}-\omega\right|_{x} \leq \frac{C_{1}}{k}
$$

and

$$
\forall x \in X \quad\left|\frac{1}{k} F_{k}^{*} g_{k}^{0}-g\right|_{x} \leq \frac{C_{2}}{k} .
$$

Moreover, $F_{k}$ is injective for large $k$ and therefore an embedding.

*Received April 7, 1999; accepted for publication May 19, 1999.

†Department of Mathematics and Computer Science, Emory University, Atlanta GA 30322, USA (davidb@mathcs.emory.edu). The author supported in part by NSF grant DMS-9796195 and by an NSF postdoctoral fellowship.

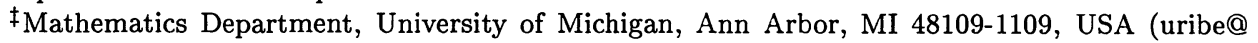
math.lsa.umich.edu). The author supported in part by NSF grant DMS-9623054.

${ }^{1}$ This convention is fairly common in complex geometry. But it is also common to include a factor of $2 \pi$, and in quantum mechanics there is yet a different convention. The choice affects our normalization of the Fubini-Study form, and will be responsible for certain factors of 2 in Sections 4 and 6. 
The precise meaning e.g. of (1.3) is: $\forall u \in T_{x} X$

$$
\left\|d F_{k}(u)\right\|_{g_{k}^{0}}^{2}=k\|u\|_{g}^{2}+O(1)
$$

where the estimate is uniform on the unit ball bundle, $\{\|u\| \leq 1\}$. The following is an easy consequence:

COROLlaRY 1.2. The $F_{k}$ are approximately pseudo-holomorphic for large $k$. More precisely:

$$
\frac{1}{k}\left\|\partial\left(F_{k}\right)_{x}\right\|=1+O\left(k^{-1}\right), \quad \text { while } \quad \frac{1}{k}\left\|\bar{\partial}\left(F_{k}\right)_{x}\right\|=O\left(k^{-1}\right),
$$

where $\|$ || denotes the operator norm, and the estimates are uniform in $x \in X$.

Theorem 1.1 says that the $F_{k}$ are projective embeddings which, for $k$ large, are both 'nearly' symplectic and 'nearly' isometric. (Recall that by a Theorem of Tischler, [15], closed integral symplectic manifolds admit symplectic embeddings into projective space, but in his proof one has no information about the isometry properties of the embedding.) In case $X$ is Kähler estimates as above were obtained by Tian, [14]. Recently, Zelditch has re-proved Tian's results using microlocal methods, [16]. Our proof of Theorem (1.1) is in part parallel to Zelditch's. However we have to work harder since we don't have the general benefits of working in the holomorphic category (e.g. $F_{k}$ is not automatically holomorphic).

The proof of Theorem 1.1 relies heavily on the machinery of Fourier integral operators of Hermite type, of Boutet de Monvel and Guillemin [8]. The family of spaces $\mathcal{H}_{k}$ are the Fourier decomposition of the image of a generalized Szegö projector $\Pi$. Theorem 1.1 is then essentially a statement about the symbol of $\Pi$.

It should also be possible to twist $L$ with a fixed Hermitian vector bundle, $\mathcal{E} \rightarrow X$, and work with generalized Szego projectors acting on the sections of a vector bundle. Just as in algebraic geometry, one then obtains embeddings into Grassmannians.

One of our goals in studying the maps $F_{k}$ is to ultimately give a microlocal proof of the recent ground-breaking theorem of Donaldson, [10], on the existence of symplectic submanifolds of a symplectic manifold that are Poincaré dual to $k[\omega]$ for $k$ large.

CONJECTURE 1.3. For large $k$ the transverse hyperplane sections of $F_{k}$ are symplectic submanifolds of $X$.

This does not follow immediately from Theorem 1.1. We need to know in addition that for large $k$ there are hyperplane sections which intersect $F_{k}(X)$ with a minimum angle $\epsilon>0$ which is independent of $k$. This is a quantitative transversality issue, just as in Donaldson's proof. His arguments however do not apply directly here: his model sections of $L^{\otimes k}$ can be said to be close to our space $\mathcal{H}_{k}$ only in an $L^{2}$ sense. In any case, we hope that the microlocal estimates on $F_{k}$ will eventually yield an independent proof.

Section 2 is devoted to preliminaries, and in $\S 3$ we review some facts about Hermite FIOs. Theorem 1.1 is proved in $\S 4$, with some details relegated to an Appendix. In $\S 5$ we give an example of the kind of weak version of Donaldson's theorem which does follow easily from Theorem 1.1. Finally, in $\S 6$ we consider the relationship between classical dynamics on $X$ and quantum dynamics on $\mathbb{P} \mathcal{H}_{k}^{*}$.

\section{Preliminaries.}


2.1. Almost-Kähler quantization. Henceforth $X, \omega, J, L, \nabla$ will be as in $\S 1$. The Hermitian metric and connection on $L$ induce corresponding structures on $L^{\otimes k}$. Together with the (fixed) metric on $X$, this defines a Laplace operator, $\Delta_{k}$, acting on $C^{\infty}\left(X, L^{\otimes k}\right)$. We now define the rescaled Laplacian,

$$
\mathcal{B}_{k}:=\Delta_{k}-n k .
$$

In [11] it was observed that Mellin's inequality implies the existence of constants $C_{1}, C_{2}>0$ such that the spectrum of $\mathcal{B}_{k}$ is contained in

$$
\left(-C_{1}, C_{1}\right) \bigcup\left(k C_{2}, \infty\right) .
$$

Therefore for large $k$ the spectrum exhibits a gap of size $O(k)$. Moreover, for large $k$ the number of eigenvalues (with multiplicities) of $\mathcal{B}_{k}$ in $\left(-C_{1}, C_{1}\right)$ is precisely equal to the value of the Riemann-Roch polynomial at $k$.

Let $Z \subset L^{*}$ denote the unit circle bundle. The connection on $L$ induces one on $Z$ (as a principal $S^{1}$ bundle), and this together with the Riemannian metric on $X$ and the standard metric on $S^{1}=\mathbb{R} / 2 \pi \mathbb{Z}$ induces a metric on $Z$ such that the projection $Z \rightarrow X$ is a Riemannian submersion with totally geodesic fibers. The Laplacian on $Z$, $\Delta_{Z}$, commutes with $\partial_{\theta}$, the infinitesimal generator of the circle action, and therefore it also commutes with the "horizontal Laplacian",

$$
\Delta_{h}:=\Delta_{Z}-\partial_{\theta}^{2} .
$$

As is well-known, the decomposition of $L^{2}(Z)$ into $S^{1}$ isotypes, $L^{2} Z=\oplus_{k} L^{2}(Z)_{k}$ is such that for each $k L^{2}(Z)_{k}$ is naturally isomorphic with $L^{2}\left(X, L^{k}\right)$. Under this isomorphism $\Delta_{h}$ gets identified with the Laplacian on sections of $L^{k}$ (induced by the metrics and the connection), and therefore the rescaled Laplacian defined above is induced by the single operator on $Z$,

$$
\mathcal{B}:=\Delta_{h}-n \partial_{\theta} .
$$

We recall the following result from [11] (see the discussion around (3.13)):

THEOREM 2.1. There exists a zeroth order self-adjoint pseudodifferential operator, $R$, on $Z$, commuting with $S^{1}$, such that the orthogonal projector

$$
\Pi: L^{2}(X) \rightarrow \mathcal{H}:=\operatorname{ker}(\mathcal{B}+R)
$$

is an Hermite $\Psi D O$ (We will recall in the next section what it means for $\Pi$ to be an Hermite $\Psi D O$.)

We can now define the spaces appearing in Theorem (1.1): Let

$$
\mathcal{H}=\widehat{\bigoplus}_{k} \mathcal{H}_{k} \quad \text { and } \quad \Pi: L^{2}(Z) \rightarrow \mathcal{H}
$$

be the decomposition of $\mathcal{H}$ under to the action of $S^{1}$. Notice that, since $R$ is of degree zero, $\mathcal{H}_{k}$ is close in $L^{2}$ to the subspace spanned by the bound-state eigenvectors of the rescaled Laplacian, for large $k$. One can naturally identify $\mathcal{H}_{k}$ as a subspace of $C^{\infty}\left(X, L^{k}\right)$. 
2.2. The coherent state map. Let $\Pi_{k}(x, y)$ denote the Schwartz kernel of the orthogonal projection,

$$
\Pi_{k}: L^{2}(Z) \rightarrow \mathcal{H}_{k}
$$

Definition 2.1. ([13]) The coherent state in $\mathcal{H}_{k}$ associated to a point $p \in Z$ is

$$
\Pi_{k}(\cdot, p) \in \mathcal{H}_{k}
$$

And the coherent state map is

$$
\begin{array}{rlc}
\Psi_{k}: Z & \rightarrow & \mathcal{H}_{k} \\
p & \mapsto & \Pi_{k}(\cdot, p)
\end{array}
$$

\section{Remarks:}

1. By their definition the coherent states have the reproducing property

$$
\forall f \in \mathcal{H}_{k}, p \in Z \quad f(p)=\left\langle\Psi_{k}(p), f\right\rangle .
$$

2. The map (2.6) clearly induces the map (that we'll denote by $\Psi_{k}^{o}$ )

$$
\begin{aligned}
\Psi_{k}^{o}: X & \rightarrow \mathbb{P} \mathcal{H}_{k} \\
x & \mapsto\left[\Psi_{k}\left(p_{x}\right)\right]
\end{aligned}
$$

where $p_{x} \in Z$ is any point projecting to $x$ and $\left[\Psi_{k}(p)\right]$ denotes the complex line through $\Psi_{k}(p)$.

3. Recall that the standard map (1.1) is defined by:

$$
F_{k}(x)=\left\{f \in \mathcal{H}_{k}: f\left(p_{x}\right)=0 \text { where } p_{x} \in P \text { satisfies } \pi\left(p_{x}\right)=x\right\}
$$

where one identifies an element of $\mathbb{P H}^{*}$ with a hyperplane in $\mathcal{H}$. Let

$$
j: \mathbb{P} \mathcal{H}_{k} \rightarrow \mathbb{P} \mathcal{H}_{k}^{*}
$$

be the (anti-holomorphic) map defined by the Hermitian metric on $\mathcal{H}_{k}$. Then by the reproducing property (2.7) the diagram

$$
\begin{array}{ccc} 
& & \mathbb{P} \mathcal{H}_{k}^{*} \\
& F_{k} & \uparrow j \\
& \stackrel{\Psi_{\text {I }}^{\circ}}{\longrightarrow} & \mathbb{P} \mathcal{H}_{k}
\end{array}
$$

commutes. Since the statements of Theorem 1.1 are invariant under composition of $F_{k}$ by $j^{-1}$, it suffices to prove the Theorem for the map $\Psi_{k}^{o}$.

\section{Hermite FIOs.}


3.1. Structure of $\Pi$. The key technical feature of the almost-Kähler quantization is the fact that the orthogonal projector $\Pi: L^{2}(Z) \rightarrow \mathcal{H}$ is an FIO of Hermite type. This means that locally we can represent its kernel as an oscillatory integral of a particular form that we now recall.

Given a manifold $M$ and a conic isotropic submanifold $\Sigma \subset T^{*} M \backslash\{0\}$, we'll denote by $J^{m}(M, \Sigma)$ the spaces of Hermite distributions introduced by Boutet de Monvel and Guillemin in [8]. In the case at hand $M=Z \times Z$ and

$$
\Sigma=\left\{\left(p, r \alpha_{p} ; p,-r \alpha_{p}\right) ; r>0, p \in Z\right\} \subset T^{*}(Z \times Z) \backslash\{0\}
$$

where $\alpha$ is the connection for on $Z$. Then

$$
\Pi \in J^{1 / 2}(Z \times Z, \Sigma) \text {. }
$$

A distribution $u \in J^{m}(Z \times Z, \Sigma)$ has the following local description. We can cover $Z \times Z$ by (finitely many) coordinate patches, so that in patch the kernel is written as an integral over phase variables $(\tau, \eta) \in \mathbb{R}_{+} \times \mathbb{R}^{2 n} \backslash\{0\}$ :

$$
u(x, y)=\int e^{i \tau f(x, y)+i \eta \cdot g(x, y)} a(x, y, \tau, \eta / \sqrt{\tau}) d \tau d \eta
$$

Here the phase functions $f$ and $g_{j}, j=1, \ldots, 2 n$, are smooth functions which "parametrize" $\Sigma$ in the sense that

$$
\Sigma=\left\{(x, y ; \tau d f(x, y)): \tau>0, f(x, y)=g_{1}(x, y)=\cdots=g_{2 n}(x, y)=0\right\} .
$$

Thus $f=g_{1}=\cdots=g_{2 n}=0$ must define the diagonal in $Z \times Z$, and $d f(x, x)=$ $\left(\alpha_{x},-\alpha_{x}\right) \in T^{*}(Z \times Z)$. The amplitude, $a(x, y, \tau, \xi)$, is polyhomogeneous in $\tau$ with decreasing half-integer powers, and rapidly decreasing as a function of $\xi$. For a distribution in $J^{m}(Z \times Z ; \Sigma)$, the leading term in the amplitude will be of the form $\tau^{m-1 / 2} a_{0}(x, y, \xi)$. An application of stationary phase shows that these requirements insure that the wave front set of (3.2) is contained in $\Sigma$.

The symbol of a distribution in $J^{m}(Z \times Z ; \Sigma)$ is the invariant object corresponding to $a_{0}(x, y, \xi)$. This object has two parts. (As usual, to get an invariant symbol one should consider distributions acting on half-forms.) The first part of the symbol is a half-form on $\Sigma$, i.e. the square-root of a top-degree differential form. (This would be the full symbol for a Lagrangian distribution.) To make the square-root globally well-defined, we need a metalinear structure on $\Sigma$.

The second part of the symbol captures the $\xi$ dependence of $a_{0}$. Let $\Sigma \subset T^{*} M \backslash\{0\}$ be an isotropic submanifold. At each point $z \in \Sigma$ we define the symplectic normal space:

$$
\mathcal{N}_{z}:=T_{z} \Sigma^{\perp} / T_{z} \Sigma
$$

and $\mathcal{N}=\bigcup_{z \in \Sigma} \mathcal{N}_{z}$ the symplectic normal bundle of $\Sigma$. Suppose we are given a metaplectic structure on $\mathcal{N}_{z}$. Then for every $z \in \Sigma$ one can construct the Heisenberg group $\mathbb{H}_{z}=\mathcal{N}_{z} \oplus \mathbb{R}$ associated with $\mathcal{N}_{z}$ and the metaplectic group $\mathcal{M}_{z}$, the double cover of the group of linear symplectic transformations of $\mathcal{N}_{z}$. Let $\mathcal{S}_{z}$ be the space of smooth vectors in the metaplectic representation of $\mathcal{M}_{z}$. The local representative $a_{0}(x, y, \xi)$ corresponds to an element of $\mathcal{S}_{z}$.

The resulting symbol is thus a smooth half-form on $\Sigma$ with values in the bundle $\mathcal{S}:=\bigcup_{z \in \Sigma} \mathcal{S}_{z}$, called a symplectic spinor. The symbol will be homogeneous with respect to the $\mathbb{R}_{+}$action on $\Sigma$, reflecting the $\tau$ dependence of the leading amplitude. 
The structures necessary to define the symbol map always exists in our case. In particular, the manifold $Z$ always possesses a metalinear structure (Lemma 2.8 of [6]). From this choice we can derive the product of a metalinear structure on $\Sigma$ and a metaplectic structure on $\mathcal{N}$.

LEMMA 3.1. The symplectic normal space to (3.1) at $z=\left(p, \alpha_{p} ; p,-\alpha_{p}\right)$ is naturally isomorphic with

$$
\mathcal{N}_{z} \cong H_{p} \oplus H_{p}
$$

where $H_{p} \subset T_{p} Z$ is the horizontal space at $p$. Therefore

$$
\mathcal{S}_{z} \cong \mathcal{S}_{p} \otimes \mathcal{S}_{p}
$$

where $\mathcal{S}_{p}$ denotes the space of smooth vectors of the metaplectic representation of the metaplectic group of $H_{p}$.

Proof. The isomorphism (3.3) is as follows. First it is clear that

$$
\mathcal{N}_{z} \cong E_{p} \oplus E_{p}
$$

where $E_{p}$ is the symplectic orthogonal to the tangent space at $\left(p, \alpha_{p}\right)$ to the symplectic submanifold

$$
\left\{\left(p, r \alpha_{p}\right) ; p \in Z, r>0\right\} \subset T^{*} Z \text {. }
$$

Then one can check that the differential of the natural projection $T^{*} Z \rightarrow Z$ induces a symplectomorphism $E_{p} \rightarrow H_{p}$ 口

Technically, in order to define the symbol of $\Pi$ invariantly we should consider $\Pi$ as an operator on half-forms. We can circumvent this issue, however, because if $\Sigma$ is as in (3.1) then one has a natural identification

$$
\Sigma \cong Z \times \mathbb{R}^{+}
$$

Under the identification $(p, r) \mapsto\left(p, r \alpha_{p}\right) \in T^{*} Z$, this space is symplectic. Therefore $\Sigma$ possesses a natural nowhere-vanishing half-form. Using this half-form and (3.4) we can conclude:

LEMMA 3.2. If $u$ is an Hermite distribution on $Z \times Z$ associated to (3.1), its symbol at $z=\left(p, \alpha_{p} ; p,-\alpha_{p}\right)$ can be naturally identified with an operator

$$
\sigma_{u}(p): \mathcal{S}_{p} \rightarrow \mathcal{S}_{p}
$$

For case of the Szegö projector (the Kähler case), the symbol of $\Pi$ was calculated in [8]. It is determined by the Kähler structure on the horizontal fibers $H_{p} \subset T_{p} Z$. The integrability of the almost complex structure plays no role in the symbol, and we get the same result for the almost-Kähler case:

Proposition 3.3. The symbol of $\Pi$ at $\left(p, \alpha_{p} ; p,-\alpha_{p}\right)$ is the rank-one projector $|e><e|$, where $e$ is the ground state of the harmonic oscillator associated to the Kähler structure of the horizontal subspace $H_{p} \subset T_{p} Z$. 
3.2. Asymptotics. Given $u \in J^{m}(Z \times Z, \Sigma)$, we can decompose into isotypes: $u=\oplus_{k} u_{k}$, where

$$
u_{k}\left(x \cdot e^{i \theta}, y\right)=e^{i k \theta} u_{k}(x, y) .
$$

The wave front set $\Sigma$ consists of a ray in the cotangent bundle over each point in the diagonal. This ray should be thought of as the "large $k$ " direction. For if $J$ is the Hamiltonian generating the $S^{1}$ action on $T^{*} Z$, we have $J\left(x, r \alpha_{x}\right)=r$. A simple wave front set argument shows that $u_{k}$ is a smooth function for each $k$. So knowledge of the singularities of $u$ may be translated into information on the large $k$ behavior of $u_{k}$.

To make this statement precise, we use the method of stationary phase.

Lemma 3.4. For $u \in J^{m}(Z \times Z, \Sigma)$, decomposed as above, there exists a constant C such that

$$
\sup _{Z \times Z}\left|u_{k}\right| \leq C k^{m+n-1 / 2}
$$

Proof. To pick off the $k$-th isotype of $u$ we average over $\theta$ :

$$
u_{k}(x, y)=\int_{0}^{2 \pi} e^{-i k \theta} u\left(x \cdot e^{i \theta}, y\right) d \theta
$$

Since $Z \times Z$ is compact, it suffices to consider the local form of $u$ given by (3.2). We will focus on the $\tau$ and $\theta$ integrations for the moment. So for the leading term we study

$$
w_{k}(x, y, \eta)=\int e^{-i k \theta} e^{i \tau f\left(x \cdot e^{i \theta}, y\right)} \tau^{m-1 / 2} a_{0}\left(x \cdot e^{i \theta}, y, \eta / \sqrt{\tau}\right) d \tau d \theta
$$

Since this expression contains local cutoffs, we can extend the $\theta$ integration to $\mathbb{R}$ to simplify the notation. Rescaling the integral by $\tau \rightarrow k \tau$ yields

$$
w_{k}(x, y, \eta)=k^{m+1 / 2} \int e^{-i k \theta+i k \tau f\left(x \cdot e^{i \theta}, y\right)} \tau^{m-1 / 2} a_{0}\left(x \cdot e^{i \theta}, y, \eta / \sqrt{k}\right) d \tau d \theta
$$

The phase function $f$ must satisfy the condition $\left.d f\right|_{(x, x)}=\left(\alpha_{x},-\alpha_{x}\right)$. Thus $\frac{d}{d \theta} f\left(x \cdot e^{i \theta}, x\right)=\alpha_{x}\left(\partial_{\theta}\right)=1$. There is freedom in the choice of $f$, so that we can require that this property hold off the diagonal as well. In fact we may as well choose $f$ so that $f\left(x \cdot e^{i \theta}, y\right)=\theta+f(x, y)$.

With this assumption, we are ready to apply stationary phase to the $\tau, \theta$ integration. The stationary point in the phase $\tau f\left(x \cdot e^{i \theta}, y\right)-\theta$ occurs at $(\tau, \theta)=(1,-f(x, y))$. Applying a stationary phase estimate with parameters, such as Theorem 7.7.6 of [12], we obtain a uniform estimate

$$
w_{k}(x, y, \eta)=C k^{m-1 / 2} e^{i k f(x, y)} a(x, y, \eta / \sqrt{k \tau})+O\left(k^{m-3 / 2}\right) .
$$

Finally, we plug this back into the $\eta$ integral and rescale to remove the $k$, which adds a power $k^{n}$. The lower order terms in the expansion of the amplitude are handled similarly.

In the above estimate, by ignoring the $\eta$ integration we did not distinguish between the behavior on and off the diagonal. If include the $\eta$ integration and restrict to a 
compact subset of $P \times P$ whose projection to $X \times X$ doesn't intersect the diagonal, then the phase has no stationary points of the phase, and we have a uniform estimate $u_{k}(x, y)=O\left(k^{-\infty}\right)$.

We also will need to understand precisely the leading behavior of $u_{k}$ on the diagonal. This could be established by an argument as above, but instead we will appeal to more general results from [8]. In this argument the stationary phase approximation is implicit in the composition theorem cited.

Lemma 3.5. For $u \in J^{m}(Z \times Z, \Sigma)$, decomposed as above, one has an asymptotic expansion

$$
u_{k}(p, p) \sim \frac{k^{n+m-\frac{1}{2}}}{(2 \pi)^{n}} \sum_{j=0}^{\infty} k^{-j / 2} f_{j}(p)
$$

as $k \rightarrow \infty$. The coefficients $f_{j}$ are smooth and the expansion is in the $C^{\infty}$ topology. Moreover, using the notation of Lemma 3.2, the leading coefficient is

$$
f_{0}(p)=\operatorname{Tr} \sigma_{u}(p)
$$

where $\operatorname{Tr} \sigma_{u}(p)$ denotes the integral along the diagonal of the Schwartz kernel of $\sigma_{u}(p)$.

If $u$ in addition has definite parity, as defined in $\S 9$ of [8], the asymptotic expansion goes down by integral powers of $k$.

Proof. The following technique has been used several times before, [6], [7]. Let $P: C^{\infty}(Z \times Z) \rightarrow C^{\infty}\left(S^{1}\right)$ be given by

$$
P u(\theta)=u\left(p \cdot e^{i \theta}, p\right),
$$

where $p$ is fixed for now, so that

$$
P u(\theta)=\sum_{k} e^{i k \theta} u_{k}(p, p)
$$

Clearly $P$ is an ordinary Fourier integral operator. One can check that the main composition theorem of [8], Theorem 7.5, applies so that $P(u)$ is a distribution on $S^{1}$ conormal to the identity. Moreover, Theorem 9.9 of [8] applies to show that $P$ preserves parity. (The excess of the composition fiber product is $2 n$, and $\operatorname{dim}(Z \times Z)-$ $2 n$ is even.) This implies the existence of the asymptotic expansions of $u_{k}(p, p)$ with the desired properties, since these are the Fourier coefficients of $P(u)$. The leading coefficient in the expansion of $u_{k}(p, p)$ is given by the symbol of $P(u)$. We defer this symbolic calculation to Appendix A.

The same argument goes through if we consider $P$ as an operator $P: C^{\infty}(Z \times$ $Z) \rightarrow C^{\infty}\left(S^{1} \times Z\right)$, so the asymptotic expansion is in the $C^{\infty}$ topology.

4. Nearly Kählerian embedding. In this section we'll prove Theorem 1.1. As noted in $\S 2$, the statements about $F_{k}$ are equivalent to those for $\Psi_{k}^{o}$. The microlocal structure described in the preceding section gives us direct estimates of the coherent state map $\Psi_{k}$, so we will work mainly with this.

We must compute the the pullback by $F_{k}$ of the Fubini-Study hermitian metric on $\mathbb{P H}_{k}$. We'll denote the pullback at the point $x \in X$ by $h_{x}(\cdot, \cdot)$, i.e.

$$
h_{x}=F_{k}^{*} g_{k}^{0}+i F_{k}^{*} \Omega_{k}
$$


To compute this, we lift tangent vectors horizontally to $T_{p} P$, where $\pi(p)=x$, pushforward by $\Psi_{k}$, and then plug the resulting vectors into the homogeneous coordinate version of the Fubini-Study hermitian metric. Letting $u_{h}, v_{h}$ denote the horizontal lifts,

$$
\frac{1}{2} h_{x}(u, v)=\frac{\left\langle d\left(\Psi_{k}\right)_{p}\left(v_{h}\right), d\left(\Psi_{k}\right)_{p}\left(u_{h}\right)\right\rangle}{\left\|\Psi_{k}(p)\right\|^{2}}-\frac{\left\langle d\left(\Psi_{k}\right)_{p}\left(v_{h}\right), \Psi_{k}(p)\right\rangle\left\langle\Psi_{k}(p), d\left(\Psi_{k}\right)_{p}\left(u_{h}\right)\right\rangle}{\left\|\Psi_{k}(p)\right\|^{4}}
$$

Here the inner products and norms are those of $\mathcal{H}_{k}$ (or equivalently $L^{2}(Z)$ ), and the normalizing factor of 2 is consistent with our choice that the curvature of the quantizing line bundle is $(-i)$ times the symplectic form. onal,

4.1. Calculation of the pullback. Let $\nu_{k}$ be the restriction of $\Pi_{k}$ to the diag-

$$
\nu_{k}(p):=\Pi_{k}(p, p)
$$

This is in fact the same as the norm square of the coherent states, by the reproducing property:

$$
\nu_{k}(p)=\left\|\Psi_{k}(p)\right\|^{2}
$$

(Incidentally, this proves that $\nu_{k}$ is strictly positive.) In the Kähler case, the function $\nu$ was introduced by Rawnsley in [13] (and further studied in [9], denoted by $\theta$ there), where it is shown that its constancy is equivalent to the coherent state map being symplectic. This doesn't hold in general, not even for all Kähler manifolds. However, we can show that $\nu_{k}$ is at least asymptotically constant, even in the almost-Kähler case.

To proceed we calculate and estimate the terms appearing in $h_{x}$.

THEOREM 4.1. For $p \in Z$, there is an asymptotic expansion of the form:

$$
\nu_{k}(p) \sim\left(\frac{k}{2 \pi}\right)^{n}+\sum_{j=1}^{\infty} k^{n-j} a_{j}(p)
$$

For $v_{h} \in T_{p} Z$ a horizontal vector, we have the asymptotic expansion:

$$
\left\langle\Psi_{k}(p), d\left(\Psi_{k}\right)_{p}\left(v_{h}\right)\right\rangle \sim \sum_{j=0} c_{j} k^{n-1 / 2-j} .
$$

And given $u, v \in T_{x} X$, which lift to the horizontal vectors $u_{h}, v_{h} \in T_{p} Z$, there is an asymptotic expansion of the form:

$$
\left\langle d\left(\Psi_{k}\right)_{p}\left(v_{h}\right), d\left(\Psi_{k}\right)_{p}\left(u_{h}\right)\right\rangle_{\mathcal{H}_{k}} \sim \frac{1}{2} \frac{k^{n+1}}{(2 \pi)^{n}}[g(u, v)+i \omega(u, v)]+\sum_{j=0}^{\infty} c_{j} k^{n-j}
$$

All three expansions are in the $C^{\infty}$ topology on the unit ball bundle of $X$.

Applying this theorem to the formula (4.1) for $h_{x}$, we see that only the first term in formula for $h_{x}$ contributes to leading order. The pullback estimates in Theorem $1.1,(1.2)$ and (1.3), then follow immediately from (4.4). 
Proof. The existence of the first asymptotic expansion (4.2) is an immediate consequence of Lemma 3.5. According to Proposition 3.3, the symbol of $\Pi$ is a rank one projector whose trace is 1 , so the leading term in the expansion is a constant which depends only on $k$ and the dimension. The factor $(k / 2 \pi)^{n}$ may be computed in a model case (the Bargmann kernel being the simplest example). The expansion is by integer steps because $\Pi$ is an operator with definite (even) parity.

We turn next to (4.4). Fix $p$ and $u_{h}, v_{h} \in T_{p} Z$ horizontal. Extend $u_{h}, v_{h}$ to two $S^{1}$-invariant horizontal vector fields on $Z, U$ and $V$ respectively.

Lemma 4.2. Consider $U_{1}$, resp. $V_{2}$, as a differential operator on $Z \times Z$ acting on the first, resp. second, variable. Then

$$
\left\langle d\left(\Psi_{k}\right)_{p}\left(v_{h}\right), d\left(\Psi_{k}\right)_{p}\left(u_{h}\right)\right\rangle_{\mathcal{H}_{k}}=\left(U_{1} \circ V_{2}\right)\left(\Pi_{k}\right)(p, p) .
$$

Proof. This follows from the reproducing property, (2.7). Let $p(s), q(t)$ be two curves on $Z$ adapted to $u, v$ respectively. Then

$$
\left\langle\Psi_{k}(q(t)), \Psi_{k}(p(s))\right\rangle=\Pi_{k}(p(s), q(t)) .
$$

Now just apply $\left.\frac{\partial^{2}}{\partial s \partial t}\right|_{(s, t)=(0,0)}$.

By the symbol calculus of Hermite distributions (precisely applying Theorem 10.2 of [8]) one gets:

$$
\left(U_{1} \circ V_{2}\right)(\Pi) \in J^{3 / 2}(Z \times Z, \Sigma)
$$

and its symbol at $\left(p, \alpha_{p} ; p,-\alpha_{p}\right)$ is known and will be described below. Notice furthermore that the right-hand side of (4.5) is precisely the $k$-th Fourier coefficient of $\left(U_{1} \circ V_{2}\right)(\Pi)$, in the sense of Lemma 3.5. Therefore (4.7) and Lemmas 3.5 and 4.2 imply the existence of the asymptotic expansion (4.4). Moreover, $\Pi$ has definite parity and the application of pseudodifferential operators preserves parity. So the steps in the expansion are integral.

To compute the leading coefficient we must first describe the symbol of $\left(U_{1}\right.$ 。 $\left.V_{2}\right)(\Pi)$. Let $H_{p} \subset T_{p} Z$ be the horizontal subspace at $p$, and recall that $u=U_{p}, v=$ $V_{p}$. Under the infinitesimal Heisenberg representation the vectors $u, v$ induce skewhermitian operators, $\tilde{u}, \tilde{v}$, on the smooth vectors of the metaplectic representation of $H_{p}, \mathcal{S}_{p}$. By Theorem 10.2 of [8] and the explicit form of the isomorphism (3.3), the symbol of (4.7) at $p$ is $|\tilde{u}(e)\rangle \otimes|\tilde{v}(e)\rangle$. We can compute this explicitly in terms of the Kähler form on on the horizontal space $H_{p}$.

LEMMA 4.3. Let $(H, h)$ be a Hermitian vector space of dimension $2 n$ and $\mathcal{F}$ a (Hilbert) representation space for the metaplectic and Heisenberg representations of $H$. Let $e \in \mathcal{F}$ be a normalized ground state of the harmonic oscillator. Then:

$$
\forall u, v \in H \quad\langle\tilde{u}(e), \tilde{v}(e)\rangle_{\mathcal{F}}=\frac{1}{2} h(u, v) .
$$

Proof. It suffices to prove it for the standard model, $H=\mathbb{C}^{n}$ with $h$ the standard hermitian form. We can take: $\mathcal{F}=L^{2}\left(\mathbb{R}^{n}\right)$ and $e=(\pi)^{-n / 4} e^{-|x|^{2} / 2}$. If $u=a+i b$ where $a, b, \in \mathbb{R}^{n}$ then $\tilde{u}=i a \cdot x+b \cdot \nabla$ and so

$$
\tilde{u}\left(e^{-|x|^{2} / 2}\right)=(i a \cdot x-b \cdot x) e^{-|x|^{2} / 2} .
$$


A simple calculation yields (4.8).

The expansion (4.4) now follows directly from Lemmas 3.5 and 4.3. The case of (4.3) is quite similar. Following Lemma 4.2, the inner product in question may be written

$$
V_{2} \Pi \in J^{1}(Z \times Z, \Sigma)
$$

By Lemma 3.5, we get an asymptotic expansion $\sum_{j} c_{j} k^{n+1 / 2-j}$. A symbolic calculation similar to that of Lemma 4.3 shows that the coefficient of the leading power, $k^{n+1 / 2}$, is zero.

4.2. $F_{k}$ is nearly pseudo-holomorphic. Corollary 1.2 is proven as follows. Let $J^{0}$ be the almost-complex structure on $\mathbb{P H}_{k}^{*}$. At each point $p \in X$ we can decompose $d\left(F_{k}\right)_{p}=\partial\left(F_{k}\right)_{p}+\bar{\partial}\left(F_{k}\right)_{p}$, where $J^{0} \circ \partial\left(F_{k}\right)_{p}=\partial\left(F_{k}\right)_{p} \circ J$ and $J^{0} \circ \bar{\partial}\left(F_{k}\right)_{p}=$ $-\bar{\partial}\left(F_{k}\right)_{p} \circ J$. Since $F_{k}$ is nearly symplectic and nearly an isometry, it is clear that it should nearly intertwine the two almost-complex structures as well.

Proposition 4.4. For $u \in T_{p} X$,

$$
\frac{1}{k}\left\|\partial\left(F_{k}\right)_{p} u\right\|^{2}=\|u\|^{2}+O\left(k^{-1}\right), \quad \frac{1}{k}\left\|\bar{\partial}\left(F_{k}\right)_{p} u\right\|^{2}=O\left(k^{-1}\right),
$$

and therefore

$$
\frac{1}{k}\left\|d\left(F_{k}\right)_{p}(J(u))-J^{0}\left(d\left(F_{k}\right)_{p}(u)\right)\right\|^{2}=O\left(k^{-1}\right)
$$

The estimates are uniform in $p$ and in $u$ on compact sets.

Proof. For notational simplicity introduce local coordinates and denote by $A$ the matrix of $d\left(F_{k}\right)_{p}$. Then $\partial\left(F_{k}\right)_{p}$ and $\bar{\partial}\left(F_{k}\right)_{p}$ can be written as

$$
A^{ \pm}=\frac{1}{2}\left[A \mp J^{0} A J\right]
$$

So we need to compute

$$
\left\|A^{ \pm} u\right\|_{g^{0}}^{2}=\frac{1}{4}\|A u\|_{g^{0}}^{2}+\frac{1}{4}\left\|J^{0} A J u\right\|_{g^{0}}^{2} \mp \frac{1}{2} g^{0}\left(A u, J^{0} A J u\right) .
$$

Applying Theorem 4.1, we find that

$$
\|A u\|^{2}=k\|u\|_{g}^{2}+O(1)
$$

and the same for $\left\|J^{0} A J u\right\|^{2}$. The remaining term is

$$
\begin{aligned}
g^{0}\left(A u, J^{0} d\left(F_{k}\right)_{p} J u\right) & =\Omega_{k}(A u, A J u) \\
& =k \omega(u, J u)+O(1) \\
& =k\|u\|_{g}^{2}+O(1)
\end{aligned}
$$


4.3. Injectivity. Theorem 4.1 tell us that $F_{k}$ is an immersion for large $k$. Consider the possibility that $F_{k}(p)=F_{k}(q)$. This means that the coherent state $\Psi_{k}(p)$ is a multiple of $\Psi_{k}(q)$. Our first step is to show that coherent states are concentrated at the base point for large $k$. So $q$ and $p$ would have to be asymptotically close together in order for the coherent states to match.

In this subsection it will be convenient to denote by $d(\cdot, \cdot)$ not only the distance function on $X$, but also its pullback to $Z$.

LeMMA 4.5. For $p, q \in Z$,

$$
\left|\Pi_{k}(p, q)\right|=\nu_{k}(p) e^{-\frac{k}{2} d(q, p)^{2}}+O\left(k^{n-1 / 2}\right),
$$

where the estimate is uniform in both $q$ and $p$.

Proof. One way to prove this is to observe that we can choose real phase functions $\theta_{k}(q, p)$ so that

$$
G(q, p)=\sum_{k} \nu_{k}(p) e^{i \theta_{k}(q, p)} e^{-\frac{k}{2} d(q, p)^{2}}
$$

is a distribution in $J^{1 / 2}(Z \times Z ; \Sigma)$ with the same symbol as $\Pi$. (By taking Fourier transforms we can write $G$ in the form (3.2), and then read off its symbol and compare it to the harmonic oscillator ground state.) Thus $\Pi-G \in J^{0}(Z \times Z ; \Sigma)$. The uniform estimate $\left|\Pi_{k}-G_{k}\right|=O\left(k^{n-1 / 2}\right)$ is obtained from Lemma 3.4, which in turn gives the estimate above.

Alternatively, one could directly argue as in Lemma 3.4. After obtaining the leading term in $\Pi$ in (3.5), one can simply compute $a_{0}(x, x, \eta)$ in local coordinates from the inverse of the symbol map.

Corollary 4.6. There is a constant $C$ such that for any sequence $x_{m}, y_{m} \in X$ such that $F_{k_{m}}\left(x_{m}\right)=F_{k_{m}}\left(y_{m}\right), k_{1}<k_{2}<\ldots$ we have

$$
d\left(x_{m}, y_{m}\right)<C k_{m}^{-3 / 4}
$$

Proof. Choose $p_{m}, q_{m}$ so that $\pi\left(p_{m}\right)=x_{m}$ and $\pi\left(q_{m}\right)=y_{m}$. By assumption $\Psi_{k_{m}}\left(p_{m}\right)=\lambda_{m} \Psi_{k_{m}}\left(q_{m}\right)$. By the reproducing property we have

$$
\nu_{k_{m}}\left(p_{m}\right)=\left|\lambda_{m}\right|^{2} \nu_{k_{m}}\left(q_{m}\right)
$$

so $\left|\lambda_{m}\right|=1+O\left(k_{m}^{-1}\right)$. By Lemma 4.5 we have

$$
\left|\lambda_{m}\right| \nu_{k_{m}}\left(p_{m}\right)=\left|\Pi_{k_{m}}\left(p_{m}, q_{m}\right)\right|=\nu_{m}\left(p_{m}\right) e^{-\frac{k_{m}}{2} d\left(p_{m}, q_{m}\right)^{2}}+O\left(k_{m}^{n-1 / 2}\right) .
$$

This implies

$$
e^{-\frac{k_{m}}{2} d\left(p_{m}, q_{m}\right)^{2}} \geq 1-C k_{m}^{-1 / 2}
$$

for some constant $C$. By taking the logarithm and adjusting $C$, we get

$$
k_{m} d\left(p_{m}, q_{m}\right)^{2}<C k_{m}^{-1 / 2}
$$

and the result follows. 
To complete the proof, consider an arbitrary geodesic $\alpha: I \rightarrow X$, parametrized by arclength. We set $\gamma=F_{k} \circ \alpha: I \rightarrow \mathbb{P} \mathcal{H}_{k}$. This will not of course be a geodesic, but we do have uniform bound on the "acceleration."

LEMma 4.7. There is a constant $C$ such that for any $\alpha, k$, if $\gamma=F_{k} \circ \alpha$ then

$$
\left\|\nabla_{\dot{\gamma}} \dot{\gamma}\right\|_{g^{0}}<C k
$$

Proof. This is a matter of counting derivatives. All terms in the expression for $\nabla_{\dot{\gamma}} \dot{\gamma}$ involve a total of 2 horizontal derivatives of $\Psi_{k}$. Let $L$ be an operator of degree $m$ given as a product of horizontal vector fields on $Z$, which acts on $Z \times Z$ in the first variable. Then $L \circ \Pi \in J^{(m+1) / 2}(Z \times Z ; \Sigma)$, and by Lemma 3.4, we have

$$
\left|\left(L \Pi_{k}\right)(p, q)\right|=O\left(k^{n+m / 2}\right),
$$

uniformly in $q$ and $p$. The $k^{n}$ is cancelled by normalization, so to estimate $\nabla_{\dot{\gamma}} \dot{\gamma}$ we just include a factor of $k^{1 / 2}$ for each derivative.

Now we put these results together.

Proposition 4.8. The map $F_{k}$ is injective for all sufficiently large $k$.

Proof. Suppose there is a sequence $x_{m}, y_{m} \in X$ such that $F_{k_{m}}\left(x_{m}\right)=F_{k_{m}}\left(y_{m}\right)$ (with $k_{1}<k_{2}<\ldots$ ). By Lemma 4.6 we know $d\left(x_{m}, y_{m}\right)=O\left(k_{m}^{-3 / 4}\right)$. For large $m$ let $\alpha_{m}: I_{m} \rightarrow X$ be the geodesic segment connecting $x_{m}$ to $y_{m}$. Since $\left\|d F_{k_{m}}\left(\dot{\alpha}_{m}\right)\right\|_{g^{0}}=$ $k_{m}^{1 / 2}+O(1)$, the curve $\gamma_{m}=F_{k_{m}} \circ \alpha_{m}$ will be a closed "lasso" of length $O\left(k_{m}^{-1 / 4}\right)$.

For each $m$, we'll choose normal coordinates on $\mathbb{P} \mathcal{H}_{k_{m}}$, centered at $F_{k_{m}}\left(x_{m}\right)$. Let $|\cdot|$ denote the Euclidean norm in these coordinates. If we draw a sphere centered at the origin and tangent to the lasso, at the point of tangency $\gamma\left(t_{m}\right)$ we have

$$
\frac{\left|\dot{\gamma}_{m}\left(t_{m}\right)\right|^{2}}{\left|\ddot{\gamma}_{m}\left(t_{m}\right)\right|}<R_{m}
$$

where $R_{m}$ is the Euclidean radius of the sphere.

With normal coordinates the FS metric $g^{0}$ is the standard Euclidean metric to within order $r^{2}$, and the length of the lasso is $O\left(k_{m}^{-1 / 4}\right)$. So in the neighborhoods of interest we can estimate the difference between the two metrics by $O\left(k_{m}^{-1 / 2}\right)$. Hence we can find a constant $C$ such that $R_{m}<C k^{-1 / 4}$, and we also have $\left|\dot{\gamma}\left(t_{m}\right)\right|=k_{m}^{1 / 2}+O(1)$. The radius of curvature estimate becomes

$$
\left|\ddot{\gamma}_{m}\left(t_{m}\right)\right|>C k_{m}^{5 / 4}
$$

The acceleration vector is $\nabla_{\dot{\gamma}} \dot{\gamma}=\ddot{\gamma}+\Gamma \dot{\gamma} \dot{\gamma}$ (omiting the indices since they are of no concern here). The Christoffel symbols can be bounded by a constant times $r$, so this can be estimated by $O\left(k_{m}^{-1 / 4}\right)$, and each $\dot{\gamma}$ is $O\left(k_{m}^{1 / 2}\right)$. Thus $\left\|\nabla_{\dot{\gamma}} \dot{\gamma}\left(t_{m}\right)\right\|_{g^{0}}=$ $\left|\ddot{\gamma}\left(t_{m}\right)\right|+O\left(k^{3 / 4}\right)$. The estimate on $\left|\ddot{\gamma}_{m}\right|$ now gives

$$
\left\|\nabla_{\dot{\gamma}} \dot{\gamma}\left(t_{m}\right)\right\|_{g^{0}}>C k_{m}^{5 / 4}
$$

contradicting Lemma 4.7. $\square$ 
5. Transverse hyperplane sections. In this section we prove the following weak analogue of a theorem of Donaldson:

Theorem 5.1. Suppose $(X, \omega)$ is real-analytic (with $\omega$ giving an integral cohomology class). Then, for sufficiently large $k$, there exist hyperplanes $W$ transverse to $F_{k}$ such that $F_{k}^{-1}(W)$ is symplectic off a codimension-one real analytic subset.

The proof is a consequence of the previous results and a simple transversality argument. We can choose the other data, $J, L, \nabla$, to be real-analytic as well. Then eigenfunctions of the Laplacian $\Delta_{k}$ will be real-analytic so the coherent states and the map $F_{k}$ will be real-analytic.

We first need some preliminaries on dihedral angles. Consider a complex $N$ dimensional Hermitian vector space, $\mathcal{V}$, let $W \subset \mathcal{V}$ be a hyperplane and $V \subset \mathcal{V}$ a complex subspace of dimension $n$. Let $w \in \mathcal{V}$ be a unit vector normal to $W$, and let $\pi_{V}: \mathcal{V} \rightarrow V$ denote orthogonal projection onto $V$. The following is a measure of the dihedral angle formed by $V$ and $W$ :

Definition 5.1. We define $\vartheta(V, W):=\left\|\pi_{V}(w)\right\|^{2}$.

Notice that $0 \leq \vartheta(V, W) \leq 1$. Also $\vartheta(V, W)=0$ iff $V \subset W$, and since $W$ is a hyperplane this occurs iff $V$ and $W$ fail to intersect transversely. And it should be clear that $\vartheta(V, W)=1$ iff $w \in V$, that is iff $W$ and $V$ intersect orthogonally.

We can consider $\vartheta$ to be a function:

$$
\vartheta: \mathcal{G}_{n} \times \mathcal{G}_{N-1} \rightarrow \mathbb{R}
$$

where $\mathcal{G}_{p}$ denotes the Grassmannian of complex $p$-dimensional subspaces of $\mathcal{V}$. As such $\vartheta$ is a real-analytic function.

In our application, we will use $\vartheta$ to measure the angle between a hyperplane $W \subset \mathbb{P} \mathcal{H}_{k}^{*}$ and the complex subspace $\partial F_{k}\left(T_{x} X\right)$. If $W$ is transverse to $F_{k}$ at $x$, then $V=T_{x}\left(F_{k}^{-1}(W)\right) \subset T_{x} X$ is a subspace of real codimension 2. In fact, if $y=F_{k}(x)$ and $w \in T_{y} \mathbb{P H}_{k}^{*}$ is a unit orthogonal to $T_{y} W$, then $V$ is the kernel of the map $A: T_{x} X \rightarrow \mathbb{C}$, given by

$$
A u=\left\langle b, d F_{k}(u)\right\rangle
$$

$A$ can be decomposed into its complex linear and antilinear parts: $A=A^{+}+A^{-}$, where

$$
A^{+} u=\left\langle b, \partial F_{k}(u)\right\rangle, \quad A^{-} u=\left\langle b, \bar{\partial} F_{k}(u)\right\rangle .
$$

Recall from [10] that $V$ is a symplectic subspace of $T_{x} X$ if $\left|A^{+}\right|<\left|A^{-}\right|$(the norm with respect to the hermitian structure on $\left.T_{x} X\right)$.

LEMMA 5.2.

$$
\begin{aligned}
& \left|A^{+}\right|^{2}=k \vartheta\left(T_{y} W, \partial F_{k}\left(T_{x} X\right)\right)+O(1), \\
& \left|A^{-}\right|^{2}=O(1)
\end{aligned}
$$

where the estimates are uniform (in $x$ ).

Proof. This is a fairly direct consequence of Proposition 4.4. The bound on $A^{-}$ is immediate. To estimate $A^{+}$, we use the fact that $\partial F_{k}$ is $k$ times an isometry up to an error of $O(1)$. 
Consider now $Y_{k}:=F_{k}(X) \subset \mathbb{P} \mathcal{H}_{k}^{*}$, with $k$ large. Define the manifold

$$
\mathcal{M}:=\left\{(y, W) \in Y \times \mathbb{P} \mathcal{H}_{k} ; y \in W\right\},
$$

where $W$ is to be thought of as a hyperplane in $\mathbb{P H}_{k}^{*}$. Consider the real-analytic projection

$$
\begin{array}{ccc}
\mathcal{M} & \rightarrow & \mathbb{P} \mathcal{H}_{k} \\
(y, W) & \mapsto & W
\end{array}
$$

Applying Sard's theorem to the projection we obtain the existence of many hyperplanes, $W$, intersecting $Y$ transversely: their complement is a set of measure zero in the image of (5.2).

Proof. [Proof of Theorem 5.1] Given any $y \in Y$ there exists a hyperplane $W_{0} \ni y$ such that $\vartheta\left(T_{y} W_{0}, \partial F_{k}\left(T_{x} X\right)\right)=1$. And we can find $W$ arbitrarily close to $W_{0}$ such that $W$ is transverse to $Y$. We can thus assume $W$ is transverse to $Y$ with angle $\vartheta\left(T_{y} W, \partial F_{k}\left(T_{x} X\right)\right)$ arbitrarily close to 1 . If $k$ is sufficiently large then by Lemma 5.2 the restriction of $\omega$ to $F_{k}^{-1}(W) \subset X$ is non-degenerate at $x$.

The determinant of the restriction of $\omega$ to $F_{k}^{-1}(W)$ is a real-analytic function, which we know to be non-zero at a point. Therefore the set of zeroes is a real-analytic subset of codimension 1 .

6. Toeplitz operators and dynamics. Let $H: X \rightarrow \mathbb{R}$ be a smooth Hamiltonian. To quantize $H$ means to associate to it a sequence of self-adjoint operators, $\left\{T_{k}^{H}\right\}$, where $T_{k}^{H}: \mathcal{H}_{k} \rightarrow \mathcal{H}_{k}$ for each $k$. As already noted in [5], following ideas of Berezin [2], one way to define $T_{k}^{H}$ is by the Toeplitz (or anti-Wick) prescription:

$$
\forall \psi \in \mathcal{H}_{k} \quad T_{k}^{H}(\psi)=\Pi_{k}(H \psi),
$$

where $\Pi_{k}$ is the orthogonal projection onto $\mathcal{H}_{k}$. Since the projector $\Pi=\oplus \Pi_{k}$ defines a Toeplitz structure in the sense of [8], the assignment $H \mapsto\left\{T_{k}^{H}\right\}$ defines a deformation quantization, [4], and the spectral estimates of [7] are valid in the present setting as well. The proofs of these statements are identical to those in the Kähler case, see $o p$. cit.

Fixing a smooth Hamiltonian $H$ and suppressing the $H$-dependence from the notation, we take $T_{k}:=T_{k}^{H}$ to be the quantum Hamiltonian corresponding to $H$. The quantum dynamics are given by the sequence of 1-parameter subgroups,

$$
\tilde{U}_{k}(t)=e^{-i k t T_{k}}: \mathcal{H}_{k} \rightarrow \mathcal{H}_{k},
$$

fundamental solution to the Schrödinger equation $i k^{-1} \psi_{t}=T_{k}(\psi)$ (where $k=1 / \hbar$ ). For each $t$ the unitary map $\tilde{U}_{k}(t)$ induces a transformation of the projective space,

$$
U_{k}(t): \mathbb{P} \mathcal{H}_{k} \rightarrow \mathbb{P} \mathcal{H}_{k},
$$

which is holomorphic and an isometry. On the other hand one has the classical Hamiltonian flow of $H$, which we will denote by $\phi_{t}: X \rightarrow X$. The question arises: to what extent are the embeddings $\Psi_{k}^{o}$ equivariant? This is the issue we examine in this section.

We begin by reviewing the Hamiltonian formulation of quantum mechanics. Consider $\mathcal{H}_{k}$ as a real symplectic vector space where the symplectic form is twice the imaginary part of the Hilbert inner product. The natural $S^{1}$ action on $\mathcal{H}_{k}$ is Hamiltonian with moment map $\|\psi\|^{2}$, and the projective space $\mathbb{P} \mathcal{H}_{k}$ can be thought of as a Marsden-Weinstein reduction of $\mathcal{H}_{k}$ with respect to this action. 
To be specific, let ${ }^{k} \tilde{\Xi}$ resp. ${ }^{k} \Xi$ denote the infinitesimal generator of the oneparameter group $\tilde{U}(t)_{k}$ resp. $U_{k}(t) .{ }^{k} \tilde{\Xi}$ is the linear vector field on $\mathcal{H}_{k}$

$$
\forall \psi \in \mathcal{H}_{k} \quad{ }^{k} \tilde{\Xi}_{\psi}=-i k T_{k} \psi
$$

which is the Hamiltonian vector field of the function

$$
\tilde{Q}_{k}(\psi)=k\left\langle T_{k} \psi, \psi\right\rangle \text {. }
$$

The Hamiltonian is invariant under the $S^{1}$ action and $U_{k}$ is the reduction of $\tilde{U}_{k}$ to $\mathbb{P H}_{k}$. In other words, the vector field ${ }^{k} \Xi$ is the Hamiltonian vector field of the function

$$
Q_{k}([\psi])=k \frac{\left\langle T_{k} \psi, \psi\right\rangle}{\langle\psi, \psi\rangle}
$$

(where $[\psi]$ denotes the complex line through $\psi \neq 0$ ) with respect to the Fubini-Study symplectic form. This is known, see [1] for example and references therein. In addition to the symplectic structure, in quantum mechanics one also has a Riemannian metric on the phase space, namely the Fubini-Study metric on $\mathbb{P} \mathcal{H}_{k}$. This has a nice physical interpretation:

LEMMA 6.1. The length of ${ }^{k} \Xi$ at the quantum state $[\psi]$ is

$$
\left|{ }^{k} \Xi_{[\psi]}\right|^{2}=2 k^{2} \frac{\left\langle T_{k}^{2} \psi, \psi\right\rangle}{\langle\psi, \psi\rangle}-2 k^{2}\left(\frac{\left\langle T_{k} \psi, \psi\right\rangle}{\langle\psi, \psi\rangle}\right)^{2},
$$

That is, up to a factor of $2 k^{2}=2 / \hbar^{2}$, it is the mean-square deviation in the observation of the energy when the quantum system is in the state $[\psi]$.

We can now state some partial results on near-equivariance of $\Psi_{k}^{o}$.

Consider the symplectic submanifold $Y_{k}:=\Psi_{k}^{o}(X)$. (Although $\Psi_{k}^{o}$ is not exactly symplectic its image is a symplectic submanifold of $\mathbb{P} \mathcal{H}_{k}$.) If $\perp_{s}$ denotes the symplectic orthogonal, one has:

$$
\forall y \in Y_{k} \quad T_{y}\left(\mathbb{P} \mathcal{H}_{k}\right)=T_{y} Y_{k} \oplus\left(T_{y} Y_{k}\right)^{\perp_{s}} .
$$

We will use the notation: $v=v^{\|}+v^{\perp_{s}}$ for the components of a vector $v$ under this decomposition. In particular, the vector field along $Y_{k},\left.{ }^{k} \Xi\right|_{Y_{k}}$, splits under this decomposition

$$
\left.{ }^{k} \Xi\right|_{Y_{k}}=\left.{ }^{k} \Xi\right|_{Y_{k}} ^{\|}+\left.{ }^{k} \Xi\right|_{Y_{k}} ^{\perp s} .
$$

We will show that the tangential component is approximately equal to $d \Psi_{k}^{o}\left(\xi_{H}\right)$ :

THEOREM 6.2. One has, uniformly,

$$
d \Psi_{k}^{o}\left(\xi_{H}\right)=\left.{ }^{k} \Xi\right|_{Y_{k}} ^{\|}+O\left(k^{-1 / 2}\right)
$$

In particular $\left.{ }^{k} \Xi\right|_{Y_{k}} ^{\|}=O\left(k^{1 / 2}\right)$. Moreover, assuming that ${ }^{k} \Xi^{\perp_{s}} \neq \overrightarrow{0}$, let $\theta_{k}(y)$ denote the angle between ${ }^{k} \Xi^{\|}$and ${ }^{k} \Xi^{\perp_{s}}$ at $y \in Y_{k}$. Then

$$
\left|\cos \left(\theta_{k}(y)\right)\right|=O\left(k^{-1 / 2}\right)
$$

uniformly on $y$. If $X$ is Kähler then $\cos \left(\theta_{k}\right)$ is identically zero. 
Before giving the proof we'll go over a few facts. Consider the inclusion map

$$
\iota: Y_{k} \hookrightarrow \mathbb{P} \mathcal{H}_{k} \text {. }
$$

The following is immediate: The Hamiltonian vector field of the restriction $\iota^{*} Q_{k}$ of $Q_{k}$ to $Y_{k}$ is the tangential component of ${ }^{k} \Xi$ with respect to the above decomposition:

$$
\Xi_{\iota^{*} Q_{k}}=\left.{ }^{k} \Xi\right|_{Y_{k}} ^{\|}
$$

The function on $X$

$$
\sigma_{T_{k}}(x):=\frac{1}{k} \Psi_{k}^{o *} Q_{k}(x)=\frac{\left\langle T_{k}\left(\Psi_{k}(p)\right), \Psi_{k}(p)\right\rangle}{\left\langle\Psi_{k}(p), \Psi_{k}(p)\right\rangle}
$$

(where $p \in Z$ sits above $x$ ) is called the Wick or covariant, [2], symbol of the operator $T_{k}$. Recall that $T_{k}$ is the level $k$ Toeplitz operator associated to a smooth Hamiltonian $H: X \rightarrow \mathbb{R}$. We will refer to $H$ as the Toeplitz symbol of $T_{k}$. form

LEMMA 6.3. As $k \rightarrow \infty$ the Wick symbol has an asymptotic expansion of the

$$
\sigma_{T_{k}} \sim H+\sum_{j=1}^{\infty} \sigma_{j} k^{-j}
$$

The asymptotics are in the $C^{\infty}$ topology. In other words, to leading order the Wick and Toeplitz symbols agree.

Proof. This is another calculation in the Hermite calculus, using exactly the same technique used in the calculation of the asymptotics of the function $\nu_{k}$ in Theorem 4.1.

Proof. [Proof of Theorem 6.2] By (6.7) the pull-back, $\xi_{k}:=\Psi_{k}^{o *}\left(\left.{ }^{k} \Xi\right|_{Y_{k}} ^{\|}\right)$is the Hamilton vector field of $k \sigma_{T_{k}}$ with respect to the symplectic form $\omega_{k}:=\Psi_{k}^{o *} \Omega_{k}$ :

$$
\left.k d\left(\sigma_{T_{k}}\right)=\omega_{k}\right\rfloor \xi_{k}
$$

and by Lemma 6.3 this is $O(k)$ with respect to the fixed Riemannian metric $g$. On the other hand, by Theorem 1.1, $\omega_{k}=k \omega+O(1)$. Therefore $\xi_{k}=O(1)$. But by Lemma $6.3 d\left(\sigma_{T_{k}}\right)=d H+O(1 / k)$. Therefore

$$
\left.k d H+O(1)=k \omega\rfloor \xi_{k}+O(1)\right\rfloor \xi_{k}
$$

and since the last term is $O(1)$ we can conclude $d H=\omega\rfloor \xi_{k}+O(1 / k)$. Therefore

$$
\xi_{H}=\xi_{k}+O(1 / k)
$$

where this estimate is with respect to the fixed Riemannian metric $g$ on $X$. Applying $d \Psi_{k}^{o}$ and using Theorem 1.1 again gives (6.5).

To prove (6.6) we will estimate the Euclidean inner product

$$
g_{k}^{0}\left({ }^{k} \Xi^{\|},{ }^{k} \Xi^{\perp s}\right)=-\Omega_{k}\left(J^{0 k} \Xi^{\|},{ }^{k} \Xi^{\perp s}\right),
$$

where $J^{0}$ is the complex structure on the real tangent bundle of $\mathbb{P} \mathcal{H}_{k}$. The idea is that $\Psi_{k}^{o}$ is nearly antiholomorphic, so $J^{0}\left({ }^{k} \Xi^{\|}\right)$is nearly tangent to $Y_{k}$. More precisely, by (4.9) we have

$$
d \Psi_{k}^{o}(\nabla H)=d \Psi_{k}^{o}\left(J \xi_{H}\right)=-J^{0} d \Psi_{k}^{o}\left(\xi_{H}\right)+O(1)
$$


Coupled with (6.5), we get:

$$
J^{0}\left({ }^{k} \Xi^{\|}\right)=-d \Psi_{k}^{o}(\nabla H)+\epsilon_{k}
$$

where $\left|\epsilon_{k}\right|=O(1)$. Since $d \Psi_{k}^{o}(\nabla H)$ is tangent to $Y_{k}$ and ${ }^{k} \Xi^{\perp_{s}}$ is in the symplectic orthogonal,

$$
g_{k}^{0}\left({ }^{k} \Xi^{\|},{ }^{k} \Xi^{\perp_{s}}\right)=-\Omega_{k}\left(\epsilon_{k},{ }^{k} \Xi^{\perp_{s}}\right)=g_{k}^{0}\left(\epsilon_{k}, J^{0}\left({ }^{k} \Xi^{\perp_{s}}\right)\right) .
$$

By the definition of $\theta_{k}$, Schwartz' inequality and the fact that $J^{0}$ preserves the metric, we get

$$
\left.\right|^{k} \Xi^{\|}||^{k} \Xi^{\perp_{s}}|| \cos \left(\theta_{k}\right)|\leq|^{k} \Xi^{\perp_{s}}|| \epsilon_{k} \mid \text {. }
$$

Recalling that $\left|\epsilon_{k}\right|=O(1)$ and that $\left.\right|^{k} \Xi^{\|} \mid=O\left(k^{1 / 2}\right)$ yields the result.

Final remarks. In several special cases (e. g. $X=\mathbb{C P}^{1}$ ) we can prove, by direct calculation, that for all $H$

$$
\left.{ }^{k} \Xi\right|_{Y_{k}} ^{\perp s}=O(1)
$$

from which the estimate

$$
d\left(\Psi_{k}^{o}\right)_{x}\left(\xi_{H}\right)={ }^{k} \Xi_{\Psi_{k}^{o}(x)}+O(1)
$$

follows. The estimate (6.11) is an infinitesimal version of the near-equivariance of the $\Psi_{k}^{o}$. (Notice that by Theorem $1.1 d \Psi_{k}^{o}\left(\xi_{H}\right)=O\left(k^{1 / 2}\right)$.) By Lemma 6.1, the issue of whether (6.10) holds is equivalent to the issue of whether

$$
\frac{\left\langle T_{k}^{2}(\Psi(p)), \Psi(p)\right\rangle}{\langle\Psi(p), \Psi(p)\rangle}-\left(\frac{\left\langle T_{k}(\Psi(p)), \Psi(p)\right\rangle}{\langle\Psi(p), \Psi(p)\rangle}\right)^{2}=\left|\left(\xi_{H}\right)_{x}\right|^{2} k^{-1}+O\left(k^{-2}\right)
$$

holds. (Here $p \in Z$ is a point above $x \in X$.) We find this to be an interesting semiclassical question: it relates the mean-square deviation of the energy, when the system is in a coherent state, to the Riemannian length of the classical Hamiltonian vector field. One can easily see that for a given $(X, \omega, J)$ the leading asymptotics of the lefthand side of (6.12) are unchanged if one modifies $T=\Pi H \Pi$ by a pseudodifferential operator of order $(-1)$. This supports the conjecture that (6.12), and therefore (6.11), hold in general. We hope to return to this issue in the near future. (It is easy to check that (6.12) holds for $X=\mathbb{C}^{n}$ with the Euclidean metric.)

\section{Appendix A. The symbolic calculation.}

We begin by briefly reviewing the symplectic linear algebra that underlies the symbolic calculation in Lemma 3.5. Let $V$ and $W$ be symplectic vector spaces, $\Gamma \subset$ $V \times W^{-}$a Lagrangian subspace and $\Sigma \subset W$ an isotropic subspace. We think of $\Gamma$ as a canonical relation from $W$ to $V ; \Gamma \circ \Sigma$ is an isotropic subspace of $V$. (For the composition $P u$ in Lemma 3.5, $\Gamma$ and $\Sigma$ are the linearization of the canonical relations of $P$ and $u$, respectively.) Define the symplectic vector space

$$
\mathcal{N}:=\Sigma^{\perp} / \Sigma
$$

A symplectic spinor on $\Sigma$ is an element of $\mathcal{S}(\mathcal{N}) \otimes \bigwedge^{1 / 2}(\Sigma)$. Since $\Gamma$ is Lagrangian, a symbol on $\Gamma$ is just a half-form in $\bigwedge^{1 / 2}(\Gamma)$. 
The general symbol map for composition of a Lagrangian distribution with a Hermite distribution is given in $\S 6$ and $\S 7$ of [8]. Here we will present a simpler composition formula which holds under two key assumptions:

$$
\Gamma \circ \Sigma \text { is Lagrangian }
$$

and

$$
\{w \in \Sigma ;(0, w) \in \Gamma\} \subset W=\{0\} .
$$

(Both assumptions will hold for the composition of $P$ with $u$.) In general, the result of composition will be a symbol on $\Gamma \circ \Sigma$. Since we assume that this space is Lagrangian, the symbol will just a half-form in $\bigwedge^{1 / 2}(\Gamma \circ \Sigma)$. Thus the symbol composition formula we are looking for will be a map

$$
\mathcal{S}(\mathcal{N}) \otimes \bigwedge^{1 / 2}(\Sigma) \otimes \bigwedge^{1 / 2}(\Gamma) \rightarrow \bigwedge^{1 / 2}(\Gamma \circ \Sigma)
$$

Of central importance in the symbol composition is the subspace

$$
U_{1}:=\left\{w \in \Sigma^{\perp} ;(0, w) \in \Gamma\right\} \subset W .
$$

We will denote by $U$ the image of $U_{1}$ in $\mathcal{N}$. From (A.3) it follows that the projection $U_{1} \rightarrow U$ is an isomorphism.

Consider

$$
\begin{array}{rcc}
\rho: \Gamma \oplus \Sigma & \rightarrow & W \\
\left((v, w), w_{1}\right) & \rightarrow & w-w_{1}
\end{array}
$$

One can show that the image of this map is exactly $U_{1}^{\perp}$, and under the assumption (A.3) its kernel is isomorphic to $\Gamma \circ \Sigma$. In other words one has an exact sequence:

$$
0 \rightarrow \Gamma \circ \Sigma \cong \operatorname{ker}(\rho) \rightarrow \Gamma \oplus \Sigma \rightarrow U_{1}^{\perp} \rightarrow 0
$$

Using this exact sequence and (A.2), we can see that $U \subset \mathcal{N}$ is a Lagrangian subspace, as follows. First note that $U$ will always be isotropic, because $\Gamma$ is isotropic. By (A.5),

$$
\operatorname{dim} U_{1}^{\perp}=\frac{1}{2}(\operatorname{dim} V+\operatorname{dim} W)+\operatorname{dim} \Sigma-\operatorname{dim} \Gamma \circ \Sigma .
$$

But $\operatorname{dim} \Gamma \circ \Sigma=\frac{1}{2} \operatorname{dim} V$ by (A.2), so

$$
\operatorname{dim} U_{1}^{\perp}=\frac{1}{2} \operatorname{dim} W+\operatorname{dim} \Sigma .
$$

And this means

$$
\operatorname{dim} U=\operatorname{dim} U_{1}=\frac{1}{2} \operatorname{dim} W-\operatorname{dim} \Sigma=\frac{1}{2} \operatorname{dim} \mathcal{N},
$$

so $U$ is Lagrangian.

We are now prepared to describe the two ingredients which yield the symbol map (A.4). 
LEMMA A.1. Under the assumption (A.3) there exists a canonical isomorphism

$$
\bigwedge^{1 / 2}(\Sigma) \otimes \bigwedge^{1 / 2}(\Gamma) \cong \bigwedge^{1 / 2}(U) \otimes \bigwedge^{1 / 2}(\Gamma \circ \Sigma)
$$

Proof. When applied to the exact sequence (A.5), the functor $\bigwedge^{1 / 2}$ gives

$$
\bigwedge^{1 / 2}(\Sigma) \otimes \bigwedge^{1 / 2}(\Gamma) \cong \bigwedge^{1 / 2}\left(U_{1}^{\perp}\right) \otimes \bigwedge^{1 / 2}(\Gamma \circ \Sigma)
$$

We also have an isomorphism $U_{1}^{\perp} \cong U \oplus\left(U_{1}^{\perp} / U_{1}\right)$. The space $U_{1}^{\perp} / U_{1}$ is symplectic and carries a natural half-form. The final result is obtained by dividing out by this form.

The second ingredient is the Kostant pairing:

LEMMA A.2. (Kostant) If $\mathcal{N}$ is a symplectic vector space and $U \subset \mathcal{N}$ a Lagrangian subspace there is a natural pairing

$$
\mathcal{S}(\mathcal{N}) \otimes \bigwedge^{1 / 2}(U) \rightarrow \mathbb{C}
$$

Proof. $U$ is a maximal abelian subalgebra of the Heisenberg group of $\mathcal{N}$, and under the Heisenberg representation the intersection of the kernels of the operators in $U$ form a 1-dimensional subspace of $H_{-\infty}$, the dual space to $\mathcal{S}(\mathcal{N})$ (space of tempered distributions). Kostant proved that this subspace is naturally isomorphic with $\bigwedge^{1 / 2}(U)$

To obtain the symbol map (A.4), apply first the map from Lemma A.1 and then the pairing from Lemma A.2.

We now finish the symbolic calculation in Lemma 3.5. The operator $P$ from (3.8) is a Fourier integral operator associated to the following canonical relation, $\Gamma \subset T^{*}\left(S^{1} \times Z \times Z\right)$ :

$$
\Gamma=\left\{\left(\theta,<\eta, \partial_{\theta}>; p \cdot e^{i \theta}, \eta ; p, \xi\right): \theta \in S^{1}, \eta, \xi \in T_{p} Z\right\} .
$$

To define the symbol of $P$, we must choose a trivialization of the half-form bundle of $\Gamma$ consistent with that chosen for $\Sigma$ in $\S 3$ (because after the half-forms are divided out we need to have $\left.P u(\theta)=u\left(p \cdot e^{i \theta}, p\right)\right)$. The symbol of $P$ is then the combination of the half-form on $T_{p}^{*} P$ derived from the metric and the half-form on $S^{1}$ determined by the standard metric. We also equip $\Gamma \circ \Sigma=T_{0}^{*} S^{1}$ with the natural half-form given by this identification.

Let $Y=\left(p ; r \alpha_{p}\right) ; p \in Z, r>0 \in T^{*} P$, a symplectic submanifold. Then from (3.1) the isotropic $\Sigma$ is the diagonal in $Y \times Y^{-}$, where the minus indicates the symplectic form has been reversed. Thus the symplectic normal $\mathcal{N}=Y^{\perp} \times\left(Y^{\perp}\right)^{-}$, and $U$ is the diagonal in this space. Since both $U$ and $\Sigma$ carry symplectic structures, they possess natural half-forms as well. In $\S 3$, we defined the symbol of $u \in J^{m}(Z \times Z ; \Sigma)$ using we are using the natural half-form on $\Sigma$.

The following shows that with these natural trivializations the half-form part of the symbol map drops out.

LEMMA A.3. In this case, the map of Lemma A.1 takes the natural half-forms on the right-hand side to the natural half-forms on the left.

Proof. We linearize the problem at the points

$$
\left(0,1 ; p, \alpha_{p} ; p,-\alpha_{p}\right) \in \Gamma, \quad\left(0,1 ; p, \alpha_{p} ; p,-\alpha_{p}\right) \in \Sigma
$$


and corresponding points in the other spaces. To avoid cluttered notation, for the remainder of this proof we'll use the same letters $\Gamma, \Sigma, \mathcal{N}, Y$, etc. to denote the tangent spaces to these spaces at the appropriate points.

Let $L=\left\{\xi \in T_{\alpha_{p}}\left(T_{p}^{*} Z\right): \xi\left(\partial_{\theta}\right)=0\right\}$. As a relation, $L$ defines a map from $Y^{\perp}$ to $Y$. That is, given $w \in Y^{\perp}$, there is a unique $v \in Y$ such that $w+v \in L$. We'll denote this map by $w \mapsto L(w)$.

Under the projection $T\left(T^{*} Z\right) \rightarrow T Z$, the space $Y^{\perp}$ projects to the horizontal subspace of $T_{p} Z$, which we'll denote by $H$. This map is a symplectomorphism. The space $L$ may also be identified with $H$, under the map $w \mapsto w+L(w)$. Define $V$ to be the span of $\left\{\partial_{\theta} ; \alpha_{p}\right\} \in T\left(T^{*} Z\right)$, a 2-dimensional symplectic vector space. Then using the map $L$, we obtain a symplectomorphism $Y \cong H \oplus V$. Thus $\Sigma$ is also identified with $H \oplus V$.

Let $\Lambda=\Gamma \circ \Sigma=T_{1}\left(T_{0}^{*} S^{1}\right)$. Then $\Gamma$ is identified with $\Lambda \oplus H \oplus H \oplus V$, using

$$
\left(\sigma, w, w^{\prime}, u\right) \mapsto\left(0, \sigma ; w+L(w)+u+\sigma \alpha_{p} ; w^{\prime}+L\left(w^{\prime}\right)-\sigma \alpha_{p}\right) \in \Gamma .
$$

Under this map, the natural half-form on $\Gamma$ is the combination of the natural halfforms on $\Lambda, H$, and $V$.

We will split the exact sequence (A.5) into 3 parts, according to these decompositions. The first, and simplest, is just

$$
0 \rightarrow \Lambda \rightarrow \Lambda \rightarrow 0 \rightarrow 0 \text {. }
$$

The second $\Lambda$ is identified with a subspace of $\Gamma \oplus \Sigma$ under $\sigma \mapsto\left(0, \sigma ; \sigma \alpha_{p} ;-\sigma \alpha_{p}\right) \oplus$ $\left(\sigma \alpha_{p} ;-\sigma \alpha_{p}\right)$. The corresponding half-form map is just the identity.

The second part consists of the horizontal spaces:

$$
0 \rightarrow 0 \rightarrow H \oplus H \oplus H \rightarrow H \oplus H \oplus H \rightarrow 0 .
$$

Here the first $H \oplus H \oplus H$ is identified with a subspace of $\Gamma \oplus \Sigma$ by

$$
(u, v, w) \mapsto(0,0 ; u+L(u) ; v+L(v)) \oplus(L(w) ; L(w)) \in \Gamma \oplus \Sigma,
$$

and the second we make the identification

$$
(u, v, w) \mapsto(L(u) ; L(u)) \oplus(v+L(v)+w ; w) \in U \oplus\left(U_{1}^{\perp} / U_{1}\right) .
$$

The resulting map is the symplectomorphism $(u, v, w) \mapsto(v-w, u-v, v)$, so the half-form map preserves the natural half-forms.

The final part of the sequence is

$$
0 \rightarrow 0 \rightarrow V \oplus V \rightarrow V \oplus V \rightarrow 0 .
$$

The first identification is

$$
(u, v) \mapsto(0,0 ; u ; 0) \oplus(v ; v) \in \Gamma \oplus \Sigma,
$$

and the second is the inclusion $V \oplus V \subset U_{1}^{\perp}$. The resulting map is $(u, v) \mapsto(u-v,-v)$, again a symplectomorphism.

To complete the proof of Lemma 3.5, consider the Kostant pairing for the case where $\mathcal{N}=H_{p} \times\left(H_{p}\right)^{-}$, and $U$ is the diagonal. The distribution associated to the natural half-form on $U$ is just a delta-function on the diagonal, so the Kostant pairing gives integration on the diagonal. If we write the element of $\mathcal{S}(\mathcal{N})$ as a map $\sigma: \mathcal{S}\left(H_{p}\right) \rightarrow \mathcal{S}\left(H_{p}\right)$, we just get the trace of $\sigma$, as claimed in Lemma 3.5. 


\section{REFERENCES}

[1] A. Ashtekar and T. Schilling, Geometrical formulation of quantum mechanics, in On Einstein's path (New York, 1996), Springer, New York, 1999, pp. 23-65.

[2] F. A. Berezin, General concept of quantization, Comm. Math. Phys., 40 (1975), pp. 153-174.

[3] N. Berline, E. Getzler, And M. Vergne, Heat Kernels and Dirac Operators, Springer Verlag, 1992.

[4] M. Bordemann, E. Meinrenken, and M. SChlichenmaier, Toeplitz quantization of Kähler manifolds and $g l(N), N \rightarrow \infty$ limits, Comm. Math. Phys., 165 (1994), pp. 281-296.

[5] D. BorTHWicK AND A. URIBE, Almost-complex structures and geometric quantization, Math. Res. Lett., 3 (1996), pp. 845-861.

[6] D. BorThWICK, T. PAUL, AND A. URIBE, Legendrian distributions with applications to relative Poincaré series, Invent. Math., 122 (1995), pp. 359-402.

[7] D. Borthwick, T. PAUL, AND A. URIBE, Semi-classical spectral estimates for Toeplitz operators, to appear in Annales de l'Institut Fourier.

[8] L. Boutet De Monvel And V. Guillemin, The Spectral Theory of Toeplitz Operators, Annals of Mathematics Studies 99, Princeton U. Press, Princeton, New Jersey, 1981.

[9] M. Cahen, S. Gutt, And J. H. Rawnsley, Quantization of Kähler manifolds I, J. of Geom. and Phys., 7 (1990), pp. 45-62.

[10] S. DonaLDSON, Symplectic submanifolds and almost-complex geometry, J. Differential Geometry, 44 (1996), pp. 666-705.

[11] V. GUILlemin AND A. URIBE, The Laplace operator on the $n$-th tensor power of a line bundle: eigenvalues which are uniformly bounded in n, Asymptotic analysis, 1 (1988), pp. 105-113.

[12] L. HöRmander, The Analysis of Linear Partial Differential Operators I, Springer-Verlag, 1990.

[13] J. H. Rawnsley, Coherent states and Kähler manifolds, Quart. J. Math. Oxford, 28:2 (1977), pp. 403-415.

[14] G. TIAN, On a set of polarized Kähler metrics on algebraic manifolds, J. Differential Geometry, 32 (1990), pp. 99-130.

[15] D. TISCHLER, Closed 2-forms and an embedding theorem for symplectic manifolds, J. Differential Geometry, 12 (1977), pp. 229-235.

[16] S. ZeLDITCH, Szegö kernels and theorem of Tian, Internat. Math. Res. Notices, 6 (1998), pp. 317-331. 